\title{
Analysis of dispersion of lumbar interlaminar epidural injectates
}

\author{
Jun-Mo Park, Hoon Jung, Oh Dae Kwon, Seong Wook Hong, and Kyung-Hwa Kwak \\ Department of Anesthesiology and Pain Medicine, School of Medicine, Kyungpook National University, Daegu, Korea
}

It is recognized that because most lumbar disc herniations occur posterior to the vertebrae, inflammation arises primarily in the anterior epidural space at the site of the disc herniation [1]. Therefore, deposition of steroids in the anterior epidural space directly at the site of inflammation has been the preferred treatment [2]. Until recently, the transforaminal approach has been considered to be the most target-specific, with the least volume necessary to reach the primary site of pathology (the anterior epidural spaces), compared to interlaminar approaches $[3,4]$. However, the transforaminal approach presents a higher risk of severe adverse events for patients. We have clearly confirmed through an imaging study, whether or not lumbar interlaminar epidural injectates are able to reach the anterior epidural space.

After informed, written consent was obtained, 43 patients who suffered from lower back pain without radiculopathy due to degenerative disc disease or internal disc disruption were enrolled in an Institutional Review Board-approved, prospective, controlled, single-blinded study. The male to female ratio was 1 : 2 , and the mean age was 61.9 years. Lower back pain in all study patients was treated with lumbar interlaminar epidural steroid injections (LIESIs). Patients with extruded disc rupture, severe spinal stenosis with neurogenic claudication, severe spondylolisthesis and history of spinal surgery or intervention, such as percutaneous vertebroplasty or kyphoplasty, were excluded in order to minimize the influence of anatomical deformities that could affect the dispersion pattern of lumbar interlaminar epidural injectates. The study patients were randomly divided into four groups (A, B, C and D) according to the total volume of injectate administered. Injectates were composed of $0.5 \%$ lidocaine, 20 mg of triamcinolone, and radiocontrast dye (Isovist-240, Schering) and were administered as follows: Group A received $5 \mathrm{ml}$ ( $3 \mathrm{ml}$ of $0.5 \%$ lidocaine, $20 \mathrm{mg}$ of triamcinolone [0.5 $\mathrm{ml}$ ], and $1.5 \mathrm{ml}$ of radiocontrast dye), Group B received $7.5 \mathrm{ml}$ (5 ml of $0.5 \%$ lidocaine, $20 \mathrm{mg}$ of triamcinolone, and $2 \mathrm{ml}$ of radiocontrast dye), and Group C received $10 \mathrm{ml}$ ( $7 \mathrm{ml}$ of $0.5 \%$ lidocaine, $20 \mathrm{mg}$ of triamcinolone, and $2.5 \mathrm{ml}$ of radiocontrast dye). To clarify the effect of position change on the dispersion of the injectates, Group D received $7.5 \mathrm{ml}$ ( $5 \mathrm{ml}$ of $0.5 \%$ lidocaine, $20 \mathrm{mg}$ of triamcinolone, and $2 \mathrm{ml}$ of radiocontrast dye) and assumed a supine position soon after the procedure until the computed tomography (CT) scan was taken. After 20 minutes, all patients were re-scanned from the upper endplate level of the L1 body to the S2 body level, using CT to a thickness of three millimeters. The physicians divided each vertebra into two compartments; an upper and a lower. One intervertebral disc was regarded as one compartment. Therefore, the total number of compartments was 16; from the L1 upper endplate level to the S1 upper body level (Fig. 1).

The results show that there were no statistical differences between Groups A, B, and C in the degree of dispersion of the injectates anteriorly and posteriorly in the epidural space $(\mathrm{P}>0.05)$ and that there were no statistical differences between Groups $\mathrm{B}$ and $\mathrm{D}(\mathrm{P}>0.05)$. However, in all of the groups, the degree of dispersion of the injectates within the posterior epidural space was greater than that within the anterior epidural space. No patient in this study experienced any infection, headache, intravascular injection, reaction to the contrast material and steroid, or subarachnoid injection.

Corresponding author: Jun-Mo Park, M.D., Department of Anesthesiology and Pain Medicine, School of Medicine, Kyungpook National University, 130, Dalgubeoldae-ro, Jung-gu, Daegu 700-705, Korea. Tel: 82-53-420-5863, Fax: 82-53-426-2760, E-mail: pjm4013@naver.com (c) This is an open-access article distributed under the terms of the Creative Commons Attribution Non-Commercial License (http:// creativecommons.org/licenses/by-nc/3.0/), which permits unrestricted non-commercial use, distribution, and reproduction in any medium, provided the original work is properly cited. 

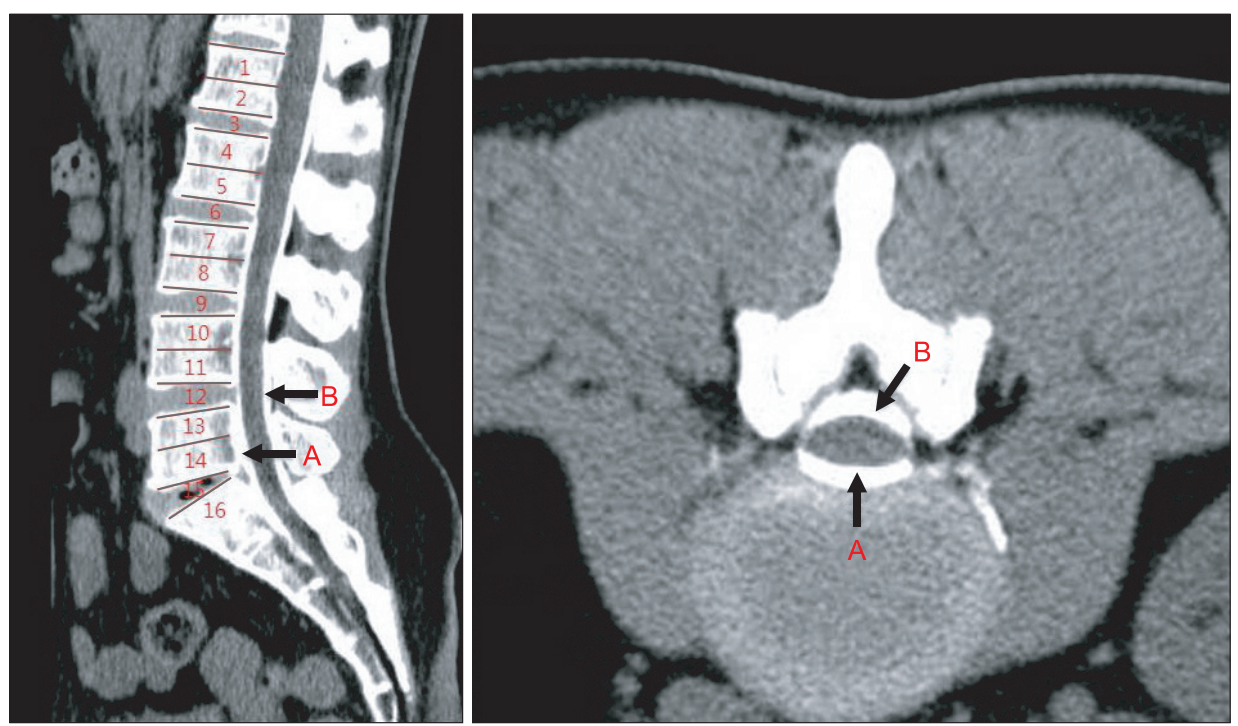

Fig. 1. Rescanned CT image taken 20 minutes after completion of the LIESI. Regions from the upper endplate of L1 to the midportion of $\mathrm{S} 1$ are divided into 16 compartments (A: anterior epidural space, B: posterior epidural space).

However, the present study has some limitations. In particular, the lack of control group to allow for comparison and assessment of the clinical effectiveness of the experimental groups is something that should be rectified in future studies. If a control group, such as an lumbar transforaminal epidural injectates (LTESIs) group, had been included in the study and the clinical effectiveness of the intervention groups had been compared with the control group, the authors would be able to determine more precisely the clinical usefulness of LIESI, although the amount of injectate that can reach the anterior epidural space would remain unknown. Second, it is possible that the small sample size may have increased the probability of type II errors (i.e. failing to detect a difference when there is one). Third, all procedures were performed by a single physician, therefore the results of the present study reflect the experience of one practitioner and may not be generalized. Although this study does have some limita- tions and flaws, to our knowledge, this is the first report verifying clearly through an imaging study, the dispersion patterns of lumbar interlaminar epidural injectates, while simultaneously considering the impact of time.

In conclusion, although the authors cannot state precisely the amount of lumbar interlaminar epidural injectate that can reach the anterior epidural space, we know that more than $5 \mathrm{ml}$ of injectate for the LIESIs can reach the anterior epidural space well. The effect of the patient's posture bears little relation to the degree of dispersion of the injectates.

\section{Acknowledgments}

The present letter was written before prohibition of triamcinolone injection into the epidural space.

\section{References}

1. Benzon HT. Epidural steroid injections for low back pain and lumbosacral radiculopathy. Pain 1986; 24: 277-95.

2. Ackerman WE 3rd, Ahmad M. The efficacy of lumbar epidural steroid injections in patients with lumbar disc herniations. Anesth Analg 2007; 104: 1217-22.

3. Botwin KP, Natalicchio J, Hanna A. Fluoroscopic guided lumbar interlaminar epidural injections: a prospective evaluation of epidurography contrast patterns and anatomical review of the epidural space. Pain Physician 2004; 7: 77-80.

4. Manchikanti L, Cash KA, Pampati V, Damron KS, McManus CD. Evaluation of lumbar transforaminal epidural injections with needle placement and contrast flow patterns: a prospective, descriptive report. Pain Physician 2004; 7: 217-23. 